\section{CANCER}

\section{Notch plays the skin field}

A recent report (Cel/ 149,1207-1220) uncovers a role for the stromal compartment in skin carcinogenesis.

Skin cancer often appears in the form of field cancerization, a constellation of alterations in the epithelial and mesenchymal tissues that surround a tumorigenic lesion, and is likely to emerge from broad exposure to carcinogens such as sunlight. However, it was not known whether stromal changes could drive this widespread transformation process.

Bing $\mathrm{Hu}$ et al. investigated the contribution of the stroma to tumorigenesis in mice by inactivating the tumor-suppressive Notch signaling pathway in mesenchymal cells. Interestingly, when they aged, these mice developed spontaneous, multifocal squamous carcinomas of diverse cellular origin, which were infiltrated by inflammatory cells. The formation of these lesions could be prevented by anti-inflammatory drug treatment.

In further studies, the authors confirmed that mouse and human skin fibroblasts with dysfunctional Notch signaling could foster the neoplastic transformation of epithelial cells by a mechanism that involved increased transcriptional activation by AP-1 factors.

Importantly, the stroma surrounding human preneoplastic skin lesions showed similar alterations in Notch signaling to those in the authors' mouse model, and these changes could be induced by exposure to ultraviolet $A$ radiation. These new results expand the protumorigenic role of the stroma that has previously been observed in other tissues such as the mammary gland and draw attention to the role of mesenchymal components as cancer drivers.-VA

\section{IMMUNOLOGY}

\section{Unexpected effects}

Foxp3 is a transcription factor that has a key role in the differentiation and function of regulatory $T\left(T_{\text {reg }}\right)$ cells. Two recent studies report that a commonly used reporter Foxp3 gene has unexpected effects in different mouse genetic backgrounds (Immunity $\mathbf{3 6}$, 717-730; Immunity 36, 731-741).

The Foxp $3^{\text {tm2 } 2 \text { Ayr }}$ allele encodes Foxp3 joined to EGFP, allowing tracking of Foxp3expressing $T_{\text {reg }}$ cells in vivo. Jaime Darce et al. found that this allele reduced the severity of disease when expressed in a mouse model of inflammatory arthritis (the $\mathrm{K} / \mathrm{BxN}$

MICROBIOLOGY

\title{
Friendly fungi
}

There's more than bacteria to the intestinal flora. According to a report in Science $(336,1314-1317)$, the gut contains indigenous fungi that interact with the immune system and may influence the course of bowel disease.

Previous studies had hinted at the existence of fungi in the gut flora. Following that lead, Iliyan Iliev et al. looked for fungal genetic material in the gut of mice and found evidence for the existence of over 100 different species.

As many fungal species interact with the innate immune receptor dectin-1, the team went on to study mice lacking this receptor

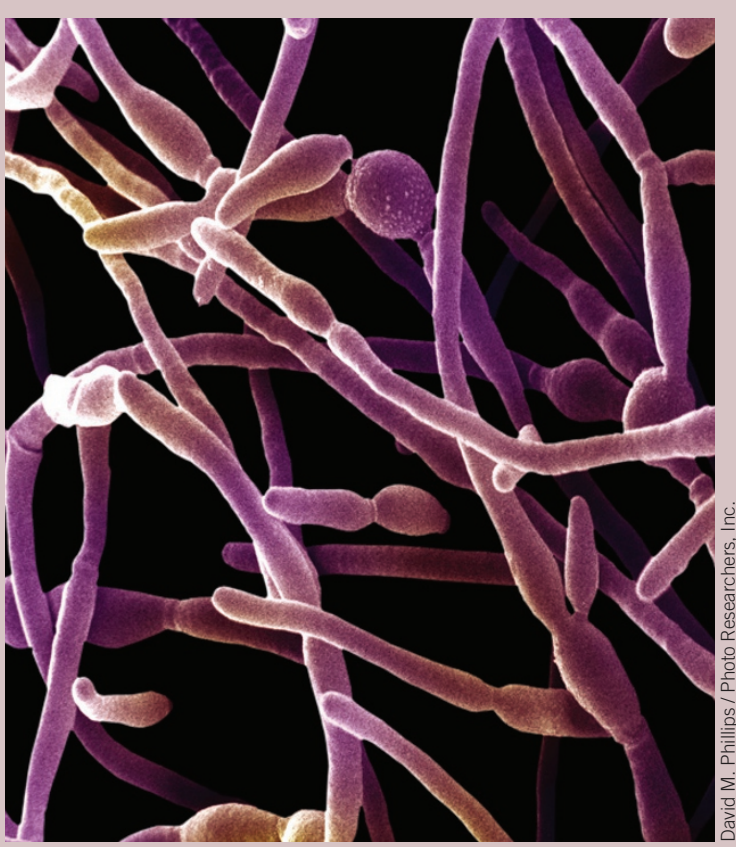
to see whether they were

differentially susceptible to inflammatory disease. In a model of chemically induced colitis, mice deficient for dectin-1 had worse disease, probably owing to an inability to keep the indigenous fungi in check.

These observations may be also relevant to humans, as the researchers identified a polymorphism in the gene for dectin-1 that is associated with a severe form of ulcerative colitis. $-J C L$

model), but made the disease worse when expressed in a nonobese diabetes (NOD) mouse model. These effects seemed to be due to the increased binding of Foxp3-EGFP to insulin regulatory factor 4 (IRF4) and lowered binding to hypoxia-inducible factor $1 \alpha$ (HIF-1 $\alpha)$, resulting in increased transcription of IRF4-regulated genes in $\mathrm{T}_{\text {reg }}$ cells and altered $T_{\text {reg }}$ cell responses. The changes in $T_{\text {reg }}$ cell responses may have differential effects on autoimmune diseases, depending on the underlying type of immunity, which may be different in diabetes and arthritis.

Similarly, Matthew Bettini et al. also observed acceleration of diabetes when Foxp $3^{t m 2 A y r}$ was expressed in a NOD model as well as impaired development of natural $T_{\text {reg }}$ cells in the thymus and of induced $T_{\text {reg }}$ cells in the periphery. They found that Foxp3-EGFP showed decreased binding to the histone acetyltransferase Tip60, histone deacetylase 7 and the transcription factor Eos. These changes altered the $T_{\text {reg }}$ transcriptome, resulting in deficient $T_{\text {reg }}$ cell responses.

These two studies show that the pres- ervation of Foxp3 interactions with other regulatory proteins is necessary for the correct transcriptional program to be enacted in $T_{\text {reg }}$ cells. Further investigations will be needed to examine whether disruption of interactions between Foxp3 and its binding partners may have affected the results of previous studies that used the Foxp $3^{\text {tm2Ayr }}$ allele.-MS

\section{CANCER}

\section{p53 functions under scrutiny}

p53 has been thought to act as tumor suppressor by arresting the cell cycle and inducing apoptosis and senescence after stress. A new study in mice shows that other activities involving metabolic regulation and antioxidant function may be key in preventing cancer and that the different p53 responses are controlled by epigenetic modifications ( $\mathrm{Ce} / \mathrm{l}$ 149, 1269-1283).

Tongyuan Li et al. mutated three different DNA acetylation sites in the Tp53 gene 